\title{
Asymptotic Bethe-Ansatz Results for a Hubbard Chain with 1/sinh-Hopping
}

\author{
P.-A. Bares ${ }^{(a)}$ and F. Gebhard ${ }^{(a, b)}$ \\ (a) Institut Laue-Langevin, B.P. 156x, F-38042 Grenoble Cedex, France \\ (b) Dept. of Physics and Materials Sciences Center, Philipps University Marburg, \\ D-35032 Marburg, Germany
}

\begin{abstract}
We investigate spin-1/2 electrons with local Hubbard interaction and variable range hopping amplitudes which decay like $\sinh (\kappa) / \sinh (\kappa r)$. Assuming integrability the Asymptotic Bethe Ansatz approach allows us to derive the generalized Lieb-Wu integral equations from the two-particle phase shift. Due to the nesting property there is a metal-to-insulator transition at $U_{c}(\kappa>0)=0^{+}$. The charge gap in the singular limit $\kappa=0$ opens when the interaction strength equals the bandwidth, $U_{c}(\kappa=0)=W>0$.
\end{abstract}

PACS1993: 71.27.+a, 71.30.+h, 05.30.Fk

Typeset using REVTEX 
Exact solutions play a crucial role in our understanding of strongly correlated systems. The algebraic and the coordinate Bethe Ansatz constitute the main line of approach to integrable systems [1]. Nevertheless, there are exactly solvable Hamiltonians whose eigenstates cannot be cast into the standard Bethe Ansatz form. A particular example is the popular Calogero-Sutherland-Moser Hamiltonian [2.3] that has attracted recently a great deal of attention in connection with the universal properties of disordered systems and random matrix theory [4,5].

Recently, Ruckenstein and one of us (F.G.) introduced a $1 / r$-Hubbard model that incorporates long-range hopping and on-site repulsion, and that includes the lattice version of the Calogero-Sutherland-Moser model in the strong-coupling limit at half band-filling [6]. The integrability as well as the structure of the eigenfunctions of this model are still poorly understood. The analysis of the two-body problem in the $1 / r$-Hubbard model indicates that the eigenstates are neither of the Bethe Ansatz form as in the Hubbard model, nor of the Jastrow-type as in the Calogero-Sutherland model [7]. Since the wave functions do not become plane waves even at large distances, Sutherland's Asymptotic Bethe Ansatz [1]:3] cannot be directly applied to the $1 / r$-Hubbard model.

In this article we introduce a model with variable range hopping that interpolates between the standard and the $1 / r$-Hubbard model. For half-filled bands and in the strong coupling limit it reduces to the antiferromagnetic $1 / \sinh ^{2}(\kappa r)$-Heisenberg or Inozemtsev model [8]. This model is a special case of an exchange interaction model [9] most recently explicitly solved in Ref. [10. The $1 / \sinh (\kappa r)$-Hubbard model is a straightforward but non-trivial generalization of the Inozemtsev model to an itinerant electron system [11].

We consider a one-dimensional Hubbard model [12] for $N$ spin-1/2 electrons with hopping amplitudes $t(l-m)$ and on-site repulsion $U$,

$$
\hat{H}=\sum_{l \neq m, \sigma} t(l-m) \hat{c}_{l, \sigma}^{+} \hat{c}_{m, \sigma}+U \sum_{l} \hat{n}_{l, \uparrow} \hat{n}_{l, \downarrow},
$$

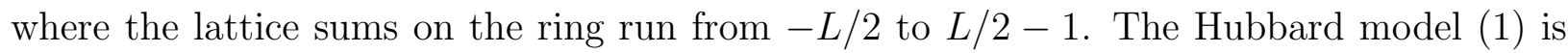
known to be exactly solvable in two cases: (i) $t(l-m)=-t \delta_{l-m, \pm 1}$ for the standard Hubbard 
model [13] and (ii) $t(l-m)=-i t(-1)^{l-m}[d(l-m)]^{-1}$ for the $1 / r$-Hubbard model [6] where $d(l-m)=(L / \pi) \sin [\pi(l-m) / L]$ denotes the chord distance between the sites $l$ and $m$. Henceforth we will use $t$ as our energy unit. Here we introduce a model on the infinite chain $(L \rightarrow \infty)$ with hopping amplitudes $t(l-m)=-i \sinh (\kappa)(-1)^{l-m} / \sinh [\kappa(l-m)]$ where $\kappa^{-1}$ controls the effective range of the hopping.

The dispersion relation $\epsilon(k)$ of our model (1) is given by $\epsilon(k)=(-2 i) \sum_{n=1}^{\infty} t(n) \sin (k n)$. It is odd under parity, and can be expressed in terms of a logarithmic derivative of thetafunctions. For $\kappa=0$ one has $\epsilon(k)=k$ with a discontinuity at the Brillouin zone boundary, while for $\kappa \rightarrow \infty$ one finds $\epsilon(k)=2 \sin k . \epsilon(k)$ is continuous for all $\kappa>0$ with zeros at $k=\pi, k=0$. The maximum (minimum) at $k=\pi / 2(k=-\pi / 2)$ for $\kappa=\infty$ is gradually shifted as function of $\kappa$ to higher (lower) momenta until it reaches $k=\pi(k=-\pi)$ for $\kappa=0$.

The corresponding low-energy ( $g$-ology [14]) Hamiltonian involves left- and right-movers with different velocities. The limit $\kappa \rightarrow 0$ is singular as the dispersion relation involves suddenly only right-movers, while the left-movers' velocity diverges like $1 / \kappa$, i.e., a discontinuity occurs in $\epsilon(k)$ at the Brillouin zone boundary. Hence the physics of the metal-to-insulator transition for $\kappa>0$ and for $\kappa=0(1 / r$-Hubbard model $)$ is completely different. At halffilling the distance $2 k_{F}=\pi n$ between the two Fermi points becomes half a reciprocal lattice vector ("perfect nesting"). The bands for right- and left-movers effectively cross each other, and their degeneracy is lifted for all $U>0$. The critical value for the transition is thus $U_{c}(\kappa>0)=0^{+}$. In the chiral $1 / r$-Hubbard model where left-movers are absent one obtains a finite critical value, $U_{c}(\kappa=0)=2 \pi$, see Ref. [6].

We discuss the symmetric orbital part $\psi\left(x_{1}, x_{2}\right)$ of the two-particle wave function in the limit of a large system with open boundary conditions. In first quantization the Schrödinger equation for the wave function $\psi\left(x_{1}, x_{2}\right)$ reads for $x_{1} \neq x_{2}$

$$
E \psi\left(x_{1}, x_{2}\right)=F\left(x_{1}, x_{2}\right)+F\left(x_{2}, x_{1}\right)+t\left(x_{1}-x_{2}\right)\left(\psi\left(x_{2}, x_{2}\right)-\psi\left(x_{1}, x_{1}\right)\right),
$$

while for $x_{1}=x_{2}$ we find $(E-U) \psi\left(x_{1}, x_{1}\right)=2 F\left(x_{1}, x_{1}\right)$. Here we defined $F\left(x_{1}, x_{2}\right)=$ $\sum_{x \neq x_{1}, x_{2}} t\left(x_{1}-x\right) \psi\left(x, x_{2}\right)$. 
We seek symmetric, spin singlet scattering solutions $\psi\left(x_{1} \ll x_{2}\right)=e^{i\left(k_{1} x_{1}+k_{2} x_{2}\right)}-$ $e^{i \theta\left(k_{1}, k_{2}\right)} e^{i\left(k_{2} x_{1}+k_{1} x_{2}\right)}$, where $\theta\left(k_{1}, k_{2}\right)$ is the phase shift, and $k_{1}, k_{2}$ are the quasi-momenta. Furthermore, we should choose $\psi\left(x_{1}, x_{2}\right)$ such that we can employ the trigonometric identity $\sinh \left(z_{1}\right) \sinh \left(z_{2}\right)=\sinh \left(z_{1}-z_{2}\right) /\left[\operatorname{coth}\left(z_{2}\right)-\operatorname{coth}\left(z_{1}\right)\right]$. This naturally leads to

$$
\begin{aligned}
\psi\left(x_{1}, x_{2}\right)= & \frac{1-\delta_{x_{1}, x_{2}}}{2 \sinh \left[\kappa\left(x_{2}-x_{1}\right)\right]}\left[e^{\kappa\left(x_{2}-x_{1}\right)}\left(A e^{i\left(k_{1} x_{1}+k_{2} x_{2}\right)}-B e^{i\left(k_{2} x_{1}+k_{1} x_{2}\right)}\right)\right. \\
& \left.-e^{-\kappa\left(x_{2}-x_{1}\right)}\left(A e^{i\left(k_{1} x_{2}+k_{2} x_{1}\right)}-B e^{i\left(k_{1} x_{1}+k_{2} x_{2}\right)}\right)\right]+\delta_{x_{1}, x_{2}} \lambda e^{i\left(k_{1}+k_{2}\right) x_{1}}
\end{aligned}
$$

as choice for the wave function $\left(B / A=e^{i \theta\left(k_{1}, k_{2}\right)}\right)$. It has precisely the form of Inozemtsev's two-magnon state [8].

The calculation of $F\left(x_{1}, x_{2}\right)$ has to be done with care because of the infinite lattice sums. For $x_{1} \neq x_{2}$ the Schrödinger equation gives $E=\epsilon\left(k_{1}\right)+\epsilon\left(k_{2}\right)$, and

$$
\lambda=(A-B)+\frac{(A+B)\left(\epsilon\left(k_{1}\right)-\epsilon\left(k_{2}\right)\right)}{2 i \sinh (\kappa)},
$$

while the equation for $x_{1}=x_{2}$ becomes $(A-B) E-i(A+B)\left(\eta\left(k_{1}\right)-\eta\left(k_{2}\right)\right)=\lambda(E-U)$ with the abbreviation $\eta(k)=2 \sinh (\kappa) \sum_{n=1}^{\infty}(-1)^{n} \cos (k n) \cosh (\kappa n) / \sinh ^{2}(\kappa n)=\eta(-k)$. The phase shift is then found as

$$
\theta\left(k_{1}, k_{2}\right)=-2 \tan ^{-1}\left[\frac{H\left(k_{2}\right)-H\left(k_{1}\right)}{U / 2}\right]
$$

with $H(k)=[-\eta(k)+\epsilon(k)(\epsilon(k)-U) /(2 \sinh (\kappa))] / 2$.

Sutherland [1,3] observed that the two-particle phase shift is sufficient to obtain the (scattering) spectrum of a model, even if the wave functions cannot explicitly be constructed as long as the model is integrable. It is far from clear that the model (11) is integrable. Nonetheless we investigate the consequences of this conjecture.

We employ periodic boundary conditions to quantize the pseudo momenta $k_{i}$. The error introduced here is exponentially small, of order $\exp (-\kappa L)$. It is clear how to set up the Asymptotic Bethe Ansatz equations for $N-M$ up-spins and $M$ down-spins in a straightforward generalization of the Lieb-Wu equations [13] because $H(k)$ plays the role of $\sin k$ : 


$$
\begin{aligned}
L k_{j} & =2 \pi I_{j}+\sum_{\beta=1}^{M} \Theta\left(2 H\left(k_{j}\right)-2 \Lambda_{\beta}\right) \\
-\sum_{j=1}^{N} \Theta\left(2 \Lambda_{\alpha}-2 H\left(k_{j}\right)\right) & =2 \pi J_{\alpha}-\sum_{\beta=1}^{M} \Theta\left(\Lambda_{\alpha}-\Lambda_{\beta}\right)
\end{aligned}
$$

for $j=1, \ldots, N ; \alpha=1, \ldots, M$, where $\Theta(x)=-2 \tan ^{-1}(2 x / U) . I_{j}$ are integers (half-odd integers) for $M$ even (odd), $J_{\alpha}$ are integers (half-odd integers) for $N-M$ even (odd).

We are interested in the ground state energy per site, $E / L$, of the model in the thermodynamic limit. In this case the equations (6) can be transformed into integral equations for the densities $\rho(k)=1 /\left[L\left(k_{j+1}-k_{j}\right)\right], \sigma(\Lambda)=1 /\left[\left(\Lambda_{\alpha+1}-\Lambda_{\alpha}\right)\right]$ in the usual way:

$$
\begin{aligned}
& 2 \pi \rho(k)=1+H^{\prime}(k) \int_{B_{1}}^{B_{2}} d \lambda \sigma(\lambda) K[H(k)-\lambda ; U / 4] \\
& 2 \pi \sigma(\lambda)=\int_{Q_{1}}^{Q_{2}} d k \rho(k) K[H(k)-\lambda ; U / 4]-\int_{B_{1}}^{B_{2}} d \lambda^{\prime} \sigma\left(\lambda^{\prime}\right) K\left[\lambda-\lambda^{\prime} ; U / 2\right]
\end{aligned}
$$

with $K[x ; y]=2 y /\left(x^{2}+y^{2}\right)$, and $H^{\prime}(k)=\partial H(k) / \partial k$. The ground state energy density is calculated as $E / L=\int_{Q_{1}}^{Q_{2}} d k \rho(k) \epsilon(k)$.

The integration limits $Q_{1}, Q_{2}, B_{1}$, and $B_{2}$ are no longer symmetric around zero. They are determined by the particle numbers, $\int_{Q_{1}}^{Q_{2}} d k \rho(k)=N / L, \int_{B_{1}}^{B_{2}} d \lambda \sigma(\lambda)=M / L$, and the condition that a charge (spin) excitation at $Q_{1}\left(B_{1}\right)$ has the same energy as the corresponding excitation at $Q_{2}\left(B_{2}\right)$. The latter conditions are most conveniently described in the pseudo particle picture [15]. The pseudo particle dispersions for charge and spin for zero external magnetic field follow from

$$
\begin{aligned}
\epsilon_{c}(k) & =\epsilon(k)+\int_{B_{1}}^{B_{2}} d \lambda \epsilon_{s}(\lambda) K[H(k)-\lambda ; U / 4] \\
2 \pi \epsilon_{s}(\lambda) & =\int_{Q_{1}}^{Q_{2}} d k H^{\prime}(k) \epsilon_{c}(k) K[H(k)-\lambda ; U / 4]-\int_{B_{1}}^{B_{2}} d \lambda^{\prime} \epsilon_{s}\left(\lambda^{\prime}\right) K\left[\lambda-\lambda^{\prime} ; U / 2\right] .
\end{aligned}
$$

The integration bounds have to fulfill $\epsilon_{c}\left(Q_{1}\right)=\epsilon_{c}\left(Q_{2}\right), \epsilon_{s}\left(B_{1}\right)=\epsilon_{s}\left(B_{2}\right)$. The ground state energy and chemical potential can then be expressed in terms of the pseudo particle energies as $E / L=\int_{Q_{1}}^{Q_{2}} d k \epsilon_{c}(k) /(2 \pi)$, and $\mu=\operatorname{Max}_{Q_{1} \leq k \leq Q_{2}} \epsilon_{c}(k)$.

For zero external magnetic field, $M=L / 2$, one finds $B_{2}=\infty=-B_{1}$. For half-filling, $N=L$, one further obtains $Q_{2}=\pi=-Q_{1}$. Therefore we can solve the integral equations analytically using Fourier transformation. With the help of the four functions 


$$
\begin{aligned}
& \widetilde{J}_{0}^{\left(\begin{array}{c}
c \\
s
\end{array}\right)}(\omega)=\left(\begin{array}{c}
\operatorname{Re} \\
\operatorname{Im}
\end{array}\right) \int_{-\pi}^{\pi} \frac{d k}{2 \pi} e^{i \omega H(k)} \\
& \widetilde{J}_{1}^{\left(\begin{array}{c}
c \\
s
\end{array}\right)}(\omega)=\left(\begin{array}{l}
\operatorname{Re} \\
\operatorname{Im}
\end{array}\right) \int_{-\pi}^{\pi} \frac{d k}{2 \pi} e^{i \omega H(k)} \epsilon(k) H^{\prime}(k) .
\end{aligned}
$$

one may express the densities and the ground state energy as

$$
\begin{aligned}
& \sigma(\lambda)=\int_{0}^{\infty} \frac{d \omega}{2 \pi \cosh (\omega U / 4)}\left[\cos (\omega \lambda) \widetilde{J}_{0}^{c}(\omega)+\sin (\omega \lambda) \widetilde{J}_{0}^{s}(\omega)\right] \\
& \rho(k)=\frac{1}{2 \pi}+H^{\prime}(k) \int_{0}^{\infty} \frac{d \omega}{\pi\left(1+e^{\omega U / 2}\right)}\left[\cos (\omega H(k)) \widetilde{J}_{0}^{c}(\omega)+\sin (\omega H(k)) \widetilde{J}_{0}^{s}(\omega)\right] \\
& E / L=\int_{0}^{\infty} \frac{2 d \omega}{1+e^{\omega U / 2}}\left[\widetilde{J}_{0}^{c}(\omega) \widetilde{J}_{1}^{c}(\omega)+\widetilde{J}_{0}^{s}(\omega) \widetilde{J}_{1}^{s}(\omega)\right] .
\end{aligned}
$$

For $\kappa \rightarrow \infty$ we have $\widetilde{J}_{0}^{s}(\omega)=0, \widetilde{J}_{0}^{c}(\omega)=J_{0}(\omega), \widetilde{J}_{1}^{c}(\omega)=-2 J_{1}(\omega) / \omega$, where $J_{0}, J_{1}$ are Bessel functions, and eq. (9c) becomes the Lieb-Wu result for the ground state energy 13 . For $\kappa \rightarrow 0$ one may rescale $\omega=z / \kappa$ which allows to perform the calculation analytically. The final result is $E / L(U \leq W)=(-W+U) / 4-U^{2} /(12 W), E / L(U \geq W)=-W^{2} /(12 U)$, with the band-width $W=2 \pi$, in complete agreement with Ref. [6]. $E / L$ is monotonically increasing as function of $U / t$ and shows no singular behavior as function of $\kappa$.

The pseudo particle dispersions become

$$
\begin{aligned}
& \epsilon_{s}(\lambda)=\int_{0}^{\infty} \frac{d \omega}{\cosh (\omega U / 4)}\left[\cos (\omega \lambda) \widetilde{J}_{1}^{c}(\omega)+\sin (\omega \lambda) \widetilde{J}_{1}^{s}(\omega)\right] \\
& \epsilon_{c}(k)=\epsilon(k)+2 \int_{0}^{\infty} \frac{d \omega}{1+e^{\omega U / 2}}\left[\cos [\omega H(k)] \widetilde{J}_{1}^{c}(\omega)+\sin [\omega H(k)] \widetilde{J}_{1}^{s}(\omega)\right] .
\end{aligned}
$$

The gap $\Delta \mu=\mu\left(n \rightarrow 1^{+}\right)-\mu\left(n \rightarrow 1^{-}\right)=U-2 \mu\left(n \rightarrow 1^{-}\right)$is given by $\Delta \mu=U-2 \operatorname{Max}\left(\epsilon_{c}(k)\right)$. For large $U / W$ one finds $\Delta \mu(U \gg W)=U-W+\mathcal{O}\left(W^{2} / U\right)$. The analytical structure of $\Delta \mu$ is very similar for all $\kappa>0$ such that the physics cannot be different from $\kappa=\infty$ where $U_{c}=0^{+}$. Consequently, $U_{c}(\kappa>0)=0^{+}$, and the gap is exponentially small for $U \ll W$. The situation changes for $\kappa=0$. Explicitly, $\epsilon_{c}(k, \kappa=0)=\left[2 k+U-\sqrt{U^{2}+W^{2}-2 W U k / \pi}\right] / 4$ such that $\Delta \mu=0$ for $U \leq W$, and $\Delta \mu=U-W$ for $U \geq W$, i.e., $U_{c}(\kappa=0)=W$, in agreement with Ref. [6] and the discussion above.

The ground state energy for strong coupling and half-filling can be cast into the form $(2 \sinh (\kappa) A=W / 2 ; \psi(x)$ is the digamma function) 


$$
\begin{aligned}
& E / L=-\frac{1}{U} \int_{-A}^{A} \frac{d x}{2 \pi} e(x) \int_{-A}^{A} d x^{\prime} \rho(x) \operatorname{Re}\left[\psi\left(\frac{1}{2}+i \frac{x-x^{\prime}}{2}\right)-\psi\left(1+i \frac{x-x^{\prime}}{2}\right)\right] \\
& e(x)=2 \sinh (\kappa)\left[\eta\left(\epsilon_{+}^{-1}(2 x \sinh (\kappa))\right)-\eta\left(\epsilon_{-}^{-1}(2 x \sinh (\kappa))\right)\right] \\
& \rho(x)=\frac{\sinh (\kappa)}{\pi}\left[\frac{1}{\epsilon^{\prime}\left(\epsilon_{+}^{-1}(2 x \sinh (\kappa))\right)}-\frac{1}{\epsilon^{\prime}\left(\epsilon_{-}^{-1}(2 x \sinh (\kappa))\right)}\right] .
\end{aligned}
$$

Here, $k_{ \pm}=\epsilon_{ \pm}^{-1}(y)$ are the two solutions of $\epsilon(k)=y$ with $\epsilon^{\prime}\left(k_{+}\right)>0, \epsilon^{\prime}\left(k_{-}\right)<0$. Numerical results for $\kappa=\infty ; 1 ; 0.1 ; 0$ are $\lim _{U \rightarrow \infty}(U E / L)=-4 \ln 2 \approx-2.773 ;-2.826 ;-3.197 ;-\pi^{2} / 3 \approx$ -3.290, respectively. The expressions (11) agree with the result obtained by Sutherland et al. [10] which supports our assumptions about integrability of the model (11). They also provide an explicit solution for the integral equations in Ref. 10 for the Inozemtsev model. We have calculated the critical exponents that control the asymptotic behavior of correlation functions by finite size scaling in conformal theory [15]. The final formulae generalize those of Ref. [15] to the case of different velocities for right- and left-moving electrons [16].

In sum, we are confident that the model (囵) is integrable and can thus be solved using the Asymptotic Bethe Ansatz. A complete construction of scattering states at finite densities is still lacking even for the $1 / \sinh ^{2}(\kappa r)$-Heisenberg model [8]. Models with variable-range exchange thus remain an interesting problem in mathematical physics.

We thank P. Nozières and A. Gogolin for stimulating discussions. F. G. thanks all his colleagues at the ILL Grenoble for their kind hospitality during a stay in 1993/94. 


\section{REFERENCES}

[1] See, e.g., Lecture Notes in Physics 242, ed. by B. S. Shastry, S. S. Jha, and V. Singh, (Springer, New York, 1985); M. A. Olshanetsky and A. M. Perelomov, Phys. Rep. 94, $313(1983)$.

[2] F. Calogero, J. Math. Phys. 10, 2191, 2197 (1969); ibid. 12, 419 (1971).

[3] B. Sutherland, J. Math. Phys. 12, 251 (1971).

[4] B. D. Simons, P. A. Lee, and B. L. Altshuler, Phys. Rev. Lett. 70, 4122 (1993); O. Narayan and B. S. Shastry, Phys. Rev. Lett. 71, 2106 (1993).

[5] F. D. M. Haldane and M. R. Zirnbauer, Phys. Rev. Lett. 71, 4055 (1993).

[6] F. Gebhard and A. E. Ruckenstein, Phys. Rev. Lett. 68, 244 (1992); F. Gebhard, A. Girndt, and A. E. Ruckenstein, Phys. Rev. B 49, 10926 (1994).

[7] F. Gebhard, Habilitation thesis, University Marburg (unpublished, 1995).

[8] V. I. Inozemtsev, Journ. Stat. Phys. 59, 1143 (1990); Comm. Math. Phys. 148, 359 (1992); V. I. Inozemtsev and B.-D. Dörfel, J. Phys. A 26, L999 (1993).

[9] A. Polychronakos, Phys. Rev. Lett. 70, 2329 (1993); B. Sutherland and B. S. Shastry, Phys. Rev. Lett. 71, p. 5 (1993), and references therein.

[10] B. Sutherland, R. A. Römer, and B. S. Shastry, Phys. Rev. Lett. 73, 2154 (1994).

[11] Compare to, e.g., V. I. Inozemtsev und A. L. Kuzemsky, Phys. Rev. B 43, 1090 (1991).

[12] J. Hubbard, Proc. R. Soc. London, Ser. A 276, 238 (1963); for a recent review on correlated electrons, see "Exactly Solvable Models of Strongly Correlated Electrons", ed. by F. H. L. Eßler and V. E. Korepin (World Scientific, Singapore, 1994).

[13] E. H. Lieb and F. Y. Wu, Phys. Rev. Lett. 20, 1445 (1968).

[14] See, e.g., J. Sólyom, Adv. Phys. 28, 201 (1979). 
[15] H. Frahm and V. E. Korepin, Phys. Rev. B42, 10553 (1990); B43, 5653 (1991).

[16] F. Gebhard and P.-A. Bares, to appear in J. Phys. Cond. Matt. (1995). 